\title{
Visualizing spin-dependent bulk scattering and breakdown of the linear dispersion relation in $\mathrm{Bi}_{2} \mathrm{Te}_{3}$
}

\author{
P. Sessi, ${ }^{1,{ }^{*}}$ M. M. Otrokov, ${ }^{2}$ T. Bathon, ${ }^{1}$ M. G. Vergniory ${ }^{3}$ S. S. Tsirkin,,${ }^{2,4}$ K. A. Kokh, ${ }^{5,6}$ O. E. Tereshchenko,,${ }^{6,7}$ \\ E. V. Chulkov, ${ }^{2,4,8,9}$ and M. Bode ${ }^{1,10}$ \\ ${ }^{1}$ Physikalisches Institut, Experimentelle Physik II, Universität Würzburg, Am Hubland, D-97074 Würzburg, Germany \\ ${ }^{2}$ Tomsk State University, prospekt Lenina 36, 634050 Tomsk, Russia \\ ${ }^{3}$ Max-Planck-Institut für Mikrostrukturphysik, D-06120 Halle, Germany \\ ${ }^{4}$ Donostia International Physics Center, Paseo de Manuel Lardizabal 4, 20018 San Sebastián/Donostia, Spain \\ ${ }^{5}$ V. S. Sobolev Institute of Geology and Mineralogy, Siberian Branch, Russian Academy of Sciences, 630090 Novosibirsk, Russia \\ ${ }^{6}$ Department of Physics, Novosibirsk State University, 630090 Novosibirsk, Russia \\ ${ }^{7}$ A. V. Rzanov Institute of Semiconductor Physics, Siberian Branch, Russian Academy of Sciences, 630090 Novosibirsk, Russia \\ ${ }^{8}$ Departamento de Física de Materiales, Universidad del País Vasco, Apartado 1072, 20080 San Sebastián/Donostia, Spain \\ ${ }^{9}$ Centro de Física de Materiales, Centro Mixto CSIC-UPVIEHU, Paseo de Manuel Lardizabal 5, 20018 San Sebastián/Donostia, Spain \\ ${ }^{10}$ Wilhelm Conrad Röntgen Center for Complex Material Systems, Universität Würzburg, Am Hubland, D-97074 Würzburg, Germany
}

(Received 4 July 2013; published 25 October 2013)

\begin{abstract}
We performed a scanning tunneling spectroscopy investigation of the electronic properties of the topological insulator $\mathrm{Bi}_{2} \mathrm{Te}_{3}$ in the energy range between -200 and $+700 \mathrm{meV}$ with respect to the Fermi level. For unoccupied states, tunneling into topological surface states dominates. Analysis of Fourier-transformed (FT) $d I / d U$ maps allowed us to obtain their energy dispersion relation and to visualize the breakdown of the linear dispersion relation typical of massless particles. For occupied states, no signature of scattering events involving surface states can be detected. FT $d I / d U$ maps reveal in this case two scattering vectors pointing along the $\overline{\Gamma M}$ and $\overline{\Gamma K}$ directions. Both are identified with scattering events within bulk states. Interestingly, vectors pointing along $\overline{\Gamma K}$ which correspond to bulk backscattering events have a length longer than the minimum distance necessary to match opposite pockets on a constant energy cut. Comparison with calculated spin-resolved constant-energy cuts shows that this is a direct consequence of the chiral spin texture present in bulk states when their energy and momentum are close to the resonances of the spin-polarized topological surface states.
\end{abstract}

DOI: 10.1103/PhysRevB.88.161407

PACS number(s): 71.70.Ej, 71.15.-m, 71.20.Nr, 73.20.-r

The recent discovery of topological insulators (TIs), a new class of materials insulating in the bulk but conducting on the surface, has opened a new route towards spintronic devices. ${ }^{1-3}$ Topological surface states of TIs are gapless and, contrary to the trivial surface states usually found at surfaces in metals and semiconductors, cannot be destroyed by the presence of defects and adsorbates as long as time-reversal symmetry is preserved. $^{4-8}$

Topological surface states are characterized by a linear energy-momentum dispersion relation, and their charge carriers can be described by a Dirac-like equation for massless particles rather than the Schrödinger equation. ${ }^{9}$ Consequently, their phase velocity is coincident with the group velocity, and carriers at different energies are all moving with same speed. Because of the Kramers degeneracy theorem, opposite spin directions are degenerate only at high-symmetry points of the surface Brillouin zone where both time- and space-reversal symmetry is preserved: the so-called Dirac points. Above and below these points the spin is perpendicularly locked to the momentum, resulting in a chiral spin texture, ${ }^{10}$ spin currents intrinsically tied to charge currents, ${ }^{11}$ and forbidden backscattering. ${ }^{12,13}$ This results in an increased spin coherence time, an important quantity for spintronic devices in which the information is encoded by the spin degree of freedom. ${ }^{14}$ In addition to possible technological applications, their spin and electronic structures make these materials a platform to search for exotic phenomena like Majorana fermions ${ }^{15}$ and magnetic monopoles. ${ }^{16}$

However, to what extent the electronic structure of topological states resembles the one typical of massless particles has not been clearly experimentally addressed so far. The same holds true concerning the spin-related properties of these materials. Spin-related effects have been investigated only for surface states, while the possible existence of similar effects for bulk states have always gone undetected.

In this Rapid Communication, we report a scanning tunneling spectroscopy (STS) study of the topological insulator $\mathrm{Bi}_{2} \mathrm{Te}_{3}$ in a large energy range, i.e., from -200 to $+700 \mathrm{meV}$ with respect to the Fermi level. We find that at positive energies tunneling into topological surface states dominates and determines the breakdown of the linear dispersion, thus experimentally setting an upper limit to the energy and momentum up to which carriers can be viewed as massless particles. At negative energies, on the other hand, Fouriertransformed (FT) $d I / d U$ maps reveal two scattering channels, both with sixfold symmetry and pointing along the $\overline{\Gamma M}$ and $\bar{\Gamma} K$ directions. Comparison with ab initio calculations shows that these vectors originate from the chiral spin texture of bulk states. Our results thus provide evidence that spin-texturerelated effects may be observed also for bulk states close to the resonances of the topological surface states.

$\mathrm{Bi}_{2} \mathrm{Te}_{3}$ single crystals were grown using the Bridgman technique. After transfer into the ultrahigh-vacuum system, samples were cleaved at room temperature at a base pressure $p=2 \times 10^{-11} \mathrm{mbar}$ and immediately inserted into a cryogenic scanning tunneling microscope operated at $T=4.8 \mathrm{~K}$. Differential conductance $(d I / d U)$ maps have been acquired simultaneously to constant-current images by means of the lock-in technique. Unless noted otherwise, all energies refer to the Fermi level. Ab initio calculations were performed 

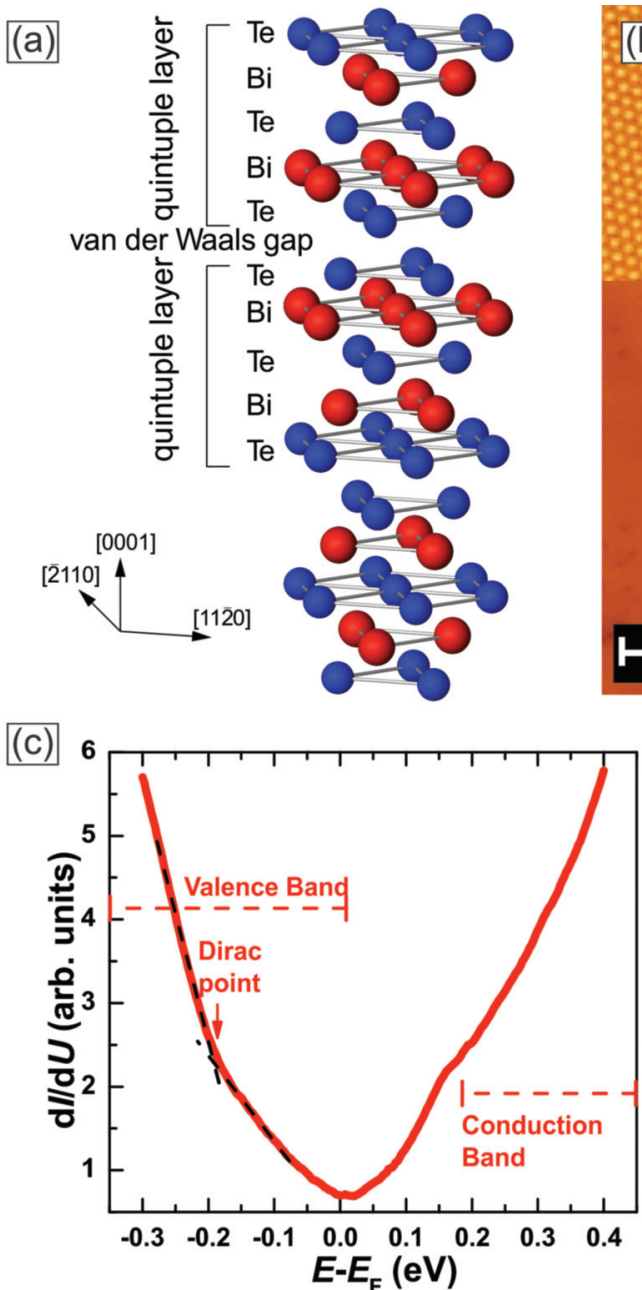
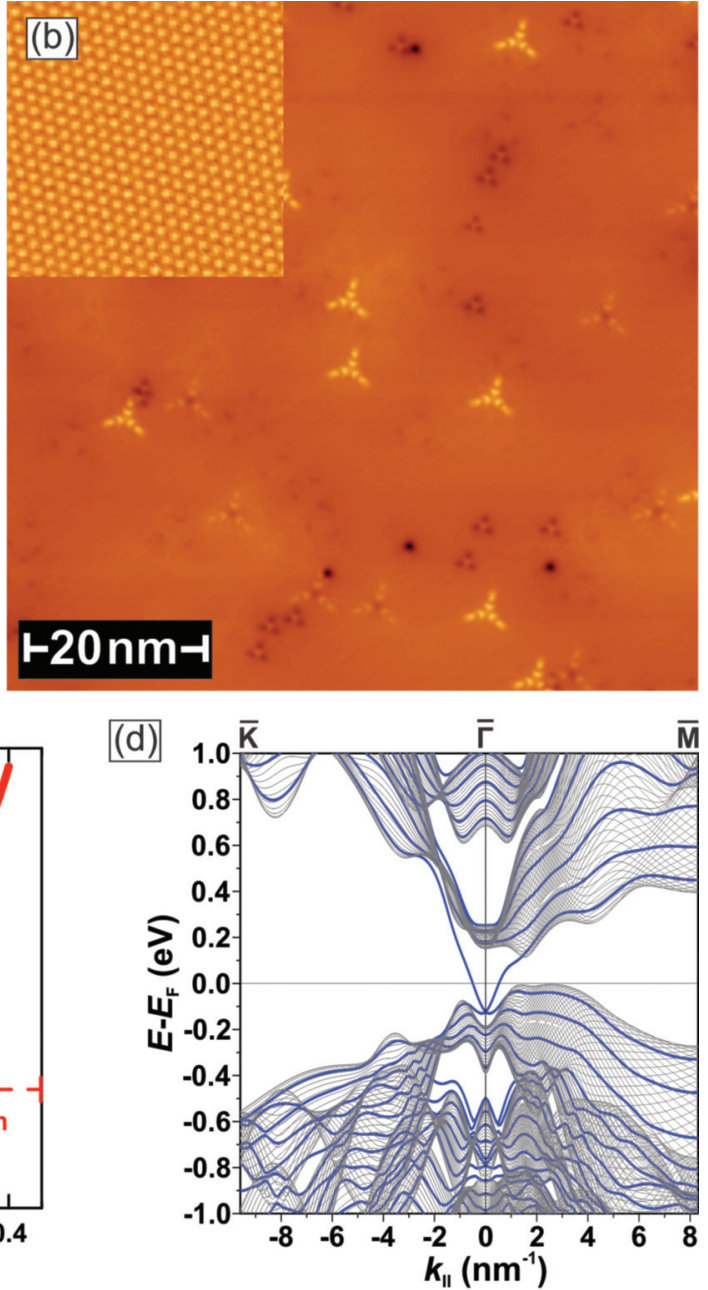

FIG. 1. (Color online) (a) $\mathrm{Bi}_{2} \mathrm{Te}_{3}$ crystal structure: alternating planes of $\mathrm{Bi}$ and $\mathrm{Te}$ are stacked on top of each other up to the formation of a quintuple layer. Quintuple layers are separated by van der Waals gaps which define the natural cleavage plane. (b) Constant-current image showing the defects present on the surface. The inset reports an atomically resolved image. (c) STS spectra and (d) calculated band structure of a 30-layer $\mathrm{Bi}_{2} \mathrm{Te}_{3}$ slab (thick blue lines) and the projected bulk band structure (thin gray lines); (b) and (c) set point: $I=50 \mathrm{pA}, U=-0.3 \mathrm{eV}$.

within the projector augmented-wave method ${ }^{17}$ in VASP implementation. ${ }^{18,19}$ The generalized gradient approximation was used to describe the exchange-correlation potential. ${ }^{20}$ The Hamiltonian contained the scalar relativistic corrections, and the spin-orbit coupling was taken into account by the second variation method. ${ }^{21}$

$\mathrm{Bi}_{2} \mathrm{Te}_{3}$ exhibits a rhombohedral structure consisting of alternating planes of $\mathrm{Bi}$ and $\mathrm{Te}$ up to the formation of a quintuple layer [Fig. 1(a)]. Quintuple layers are weakly bound by van der Waals forces, thus offering a natural cleavage plane. Figure 1(b) shows a constant-current image of $\mathrm{Bi}_{2} \mathrm{Te}_{3}$, with the inset reporting the atomically resolved structure. Different defects are present on the surface, whose origin can be understood based on their symmetry, spatial extension, and electronic character, i.e., donor or acceptor, as shown in Ref. 22. The precise classification of defects is beyond the scope of the present paper and is not important for the understanding of the intrinsic electronic properties of the host material. The electronic structure can be investigated by analyzing the local density of states as experimentally inferred by STS. Figure 1(c) shows a typical STS spectrum obtained on the pristine $\mathrm{Bi}_{2} \mathrm{Te}_{3}$ far away from any defect. The valence-band maximum and conduction-band minimum have been assigned according to the procedure presented in Ref. 23 . Although the shoulder at positive energy is less pronounced than in Ref. 23 and shifted due to different sample doping, the valence-band maximum and the conduction-band minimum can still be identified by the minimum in the $d I / d U$ curve and the shoulder at $180 \mathrm{meV}$, respectively. The position of the Dirac point is identified by the change of slope visible in the spectrum at about $-190 \mathrm{meV}$ below the Fermi level and is consistent with the fitting of the experimentally obtained linear dispersion relation (see below). Experimental data are in agreement with the results of $a b$ initio calculations presented in Fig. 1(d) within a rigid shift of about $50 \mathrm{meV}$.

To investigate the energy dispersion relation and scattering events above and below the Dirac point, we have performed quasiparticle interference (QPI) experiments in the energy range between -200 and $+700 \mathrm{meV}$. QPI mapping makes use of the standing-wave pattern generated by elastic scattering of electronic states at surface defects, such as vacancies, adsorbates, impurities, or step edges. ${ }^{24}$ Momentum 

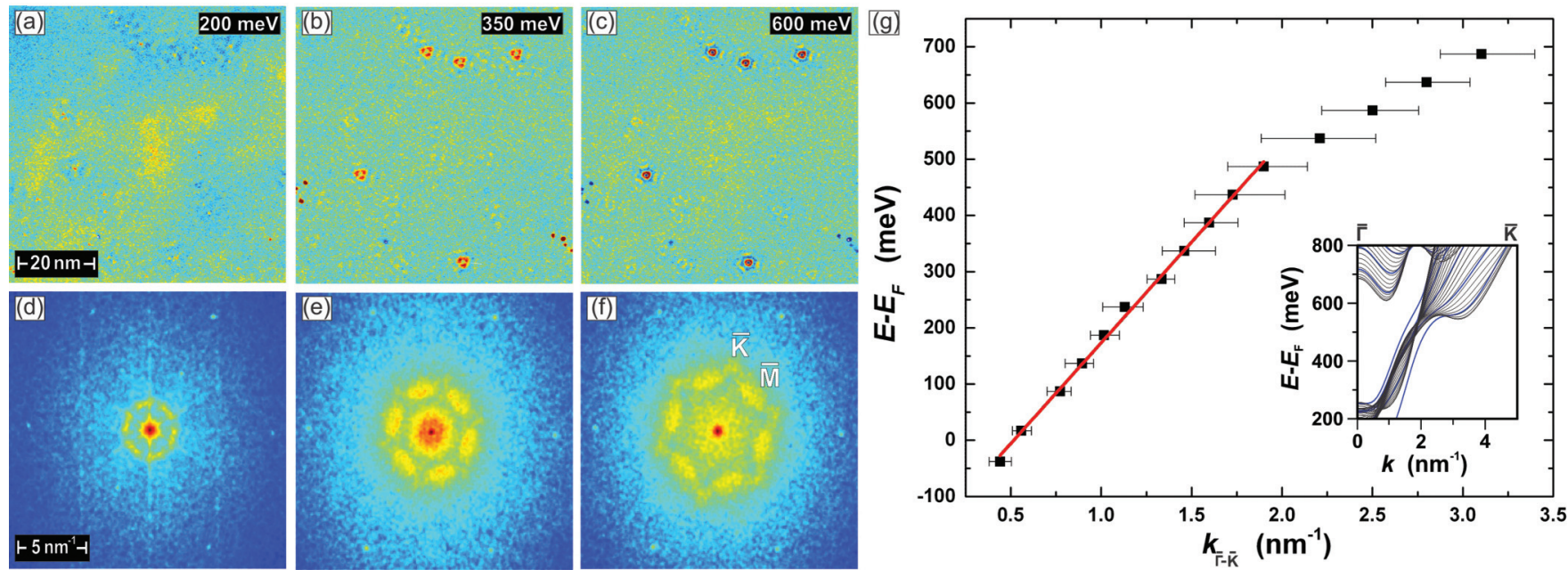

FIG. 2. (Color online) (a)-(c) $d I / d U$ maps and (d)-(f) corresponding FT $d I / d U$ maps obtained within the empty electronic states. A scattering vector with sixfold symmetry pointing along the $\overline{\Gamma M}$ direction can be seen. It is related to scattering of surface states and becomes visible for energies where the hexagonal warping already developed. (g) Energy dispersion relation of the topological surfaces state. For energies higher than $+500 \mathrm{meV}$ with respect to the Fermi level the linear dispersion relation typical of Dirac fermions breaks down, in agreement with the ab initio calculations reported in the inset. Tunneling current: $50 \mathrm{pA}$ for all images.

conservation requires $\mathbf{k}_{\mathrm{f}}=\mathbf{k}_{\mathrm{i}}+\mathbf{q}$, where $\mathbf{k}_{\mathrm{i}}$ and $\mathbf{k}_{\mathrm{f}}$ are the wave vectors of initial and final states on a constant-energy cut and $\mathbf{q}$ is the scattering vector connecting them. When initial and final states are nonorthogonal, they interfere and give rise to a spatial modulation of the local density of states characterized by a spatial periodicity equal to $2 \pi /|\mathbf{q}|{ }^{25}$ These oscillations are imaged by energy-resolved $d I / d U$ maps. Fourier transformation translates the real-space information into reciprocal space, thereby providing direct insight into scattering processes within the surface Brillouin zone. ${ }^{26}$

FT $d I / d U$ maps have been symmetrized according to the underlying lattice symmetry. Six weak spots which do not have any dispersion are visible close to the border of the images. They are due to experimental noise and thus do not have any importance for the following discussion.

At positive energies, FT $d I / d U$ maps show a scattering vector with sixfold symmetry oriented along the $\overline{\Gamma M}$ direction (Fig. 2), whose origin can be explained considering the hexagonal warping of the surface states. ${ }^{27,28}$ By analyzing its length as a function of the energy the linear dispersion relation typical of topological states can be obtained as shown in Ref. 13. The linear dispersion relation has important consequences for transport and electrostatic properties, like the defect screening length ${ }^{29}$ and the electrical tunability of the resistance through the field effect. ${ }^{30}$ It is therefore important to determine at which energy and momentum this linear dispersion relation breaks down. Since this is expected to be above the Fermi level [Fig. 1(d)], this important information cannot be extracted from photoemission experiments, which can only detect occupied states, a restriction that does not apply to STS. Indeed, the dispersion relation displayed in Fig. 2(g) shows a linear behavior up to about $500 \mathrm{meV}$ above the Fermi level. Fitting provides a Dirac point located at $E_{\mathrm{D}}=-185 \pm$ $13 \mathrm{meV}$ and a carrier velocity $v_{\mathrm{F}}=5.2 \pm 0.1 \times 10^{5} \mathrm{~m} / \mathrm{s}$. Both values are in agreement with previously published data. ${ }^{13,31}$ In contrast, at energies $E>500 \mathrm{meV}$ a strong deviation from a linear dispersion relation occurs, in agreement with the $a b$ initio calculations reported in the inset in Fig. 2(g).

This picture drastically changes at negative energies. Figures 3(a)-3(d) report $d I / d U$ maps with their corresponding FT obtained within the same area shown in Fig. 1(b) at energies of $-50,-150,-200$, and (d) $-300 \mathrm{meV}$, respectively. FT maps at $-50,-150$, and $-200 \mathrm{meV}$ in Figs. 3(e)-3(g), respectively, show three different scattering vectors, namely, $\mathbf{q}_{1}, \mathbf{q}_{2}$, and $\mathbf{q}_{3}$. Vectors $\mathbf{q}_{1}$ and $\mathbf{q}_{3}$ have an elliptical shape and are oriented along the $\overline{\Gamma M}$ direction. $\mathbf{q}_{2}$ is shorter, has an almost circular shape, and can be found in the $\bar{\Gamma} K$ direction. At $-300 \mathrm{meV}$ in Fig. 3(h), only $\mathbf{q}_{3}$ is visible.

To shed light on the origin of these vectors, experimental data have been compared with constant-energy cuts calculated $a b$ initio [Figs. 4(a)-4(c)]. They reveal elliptically shaped bulk pockets with a sixfold symmetry oriented along the $\bar{\Gamma} M$ direction and surrounding the Dirac cone. The inner side of each pocket (small-k edge close to the $\bar{\Gamma}$ point) has a chiral spin texture, which is inherited from the spin-polarized resonance of the topological surface state, merging into the bulk continuum at approximately $-75 \mathrm{meV}$ and $0.7 \mathrm{~nm}^{-1}$. Indeed, as can be seen from Figs. 4(a)-4(c), the chirality of the spin texture of the bulk pockets resembles that of the lower part of the topological surface state. As a result of the chiral spin texture, scattering between opposite pockets is strongly suppressed. Only when moving to the outer side of the pockets (large-k edge) does the spin polarization vanish, thereby allowing backscattering. Indeed, quantitative comparison with the FT maps reported in Fig. 3 reveals that the observed scattering vector $\mathbf{q}_{1}$ corresponds to backscattering of nonpolarized states at the large-k edge of opposite bulk pockets. This is not the case for the vector connecting next-nearest-neighbor bulk pockets, which is oriented along $\overline{\Gamma K}$. In this case the spin direction is not orthogonal, and consistent with the experimental data, the minimum distance matches the length of $\mathbf{q}_{2}$. 



FIG. 3. (Color online) (a)-(d) $d I / d U$ maps and (e)-(h) corresponding FT $d I / d U$ maps obtained at $-50,-150,-200$, and $-300 \mathrm{meV}$ with respect to the Fermi level. Two scattering vectors $\left(\mathbf{q}_{1}\right.$ and $\mathbf{q}_{2}$ ) are visible. Both correspond to scattering processes involving bulk states. $\mathbf{q}_{3}$ is ascribed to the FT of the scattering potential caused by the defects themselves. Tunneling current: $50 \mathrm{pA}$ for all images.

The different shapes of the scattering vectors, i.e., elliptical for $\mathbf{q}_{1}$ and circular for $\mathbf{q}_{2}$, can be explained by geometrical considerations presented in Figs. 4(d) and 4(e), which show how the scattering events observed in FT $d I / d U$ maps correspond to the filled area obtained by the intersection of two constant-energy cuts taken at the same energy and translated with respect to each other by the scattering vector. ${ }^{32}$

We would like to emphasize that, contrary to previous measurements on $\mathrm{Bi}_{2} \mathrm{Se}_{3},{ }^{33}$ no features involving surface states

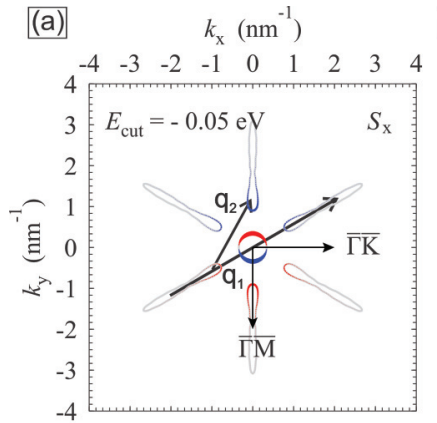

(b) $k_{\mathrm{x}}\left(\mathrm{nm}^{-1}\right) \quad$ (c) $k_{\mathrm{x}}\left(\mathrm{nm}^{-1}\right)$ (d)



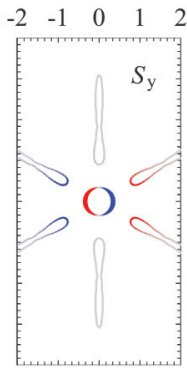

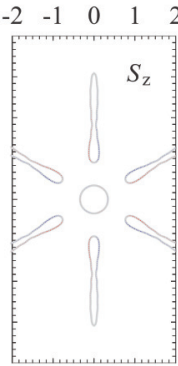

(e)

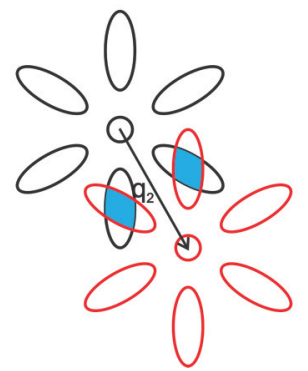

FIG. 4. (Color online) (a)-(c) Calculated constant energy cut at $-50 \mathrm{meV}$ with spin resolution represented by projections of the spin vector $\vec{S}$ on Cartesian axes. The spin vector components shown are sums of those within the Wigner-Seitz spheres around each atom in the surface quintuple layer block. Positive (negative) values of the spin vector components are shown in red (blue), while the size of the circle reflects the component absolute value. (d) and (e) Geometrical construction of scattering events: the filled area obtained by the intersection of two constant-energy cuts translated by vectors $\mathbf{q}_{1}$ and $\mathbf{q}_{2}$ represents possible scattering events and explains the anisotropic intensity observed in FT $d I / d U$ maps.

have been detected close to the Dirac point for $\mathrm{Bi}_{2} \mathrm{Te}_{3}$. In Ref. 33 features in the FT $d I / d U$ maps oriented along the $\bar{\Gamma} \bar{M}$ direction were interpreted as direct evidence of scattering from surface into bulk states. However, the data presented in Ref. 33, although taken over a wide energy range, do not show any dispersion. Furthermore, these scattering events are claimed for energies down to $-400 \mathrm{meV}$, i.e., far below the energy range where the surface state exists. As discussed above, our FT $d I / d U$ maps also provide a vector, $\mathbf{q}_{3}$ in Fig. 3, oriented along the $\bar{\Gamma} M$ direction.

We attributed the origin of this vector to the triangular defects visible in the $d I / d U$ maps. One factor often neglected in the interpretation of QPI experiments is that the signal not only depends on the initial and final states but also may be strongly influenced by the symmetry and extension of defects. In the joint-density-of-states (JDOS) approximation, ${ }^{32}$ the observed signal is given by $\operatorname{JDOS}(E, q)=V(q) \int \rho(E, k) \rho(E, k+q) d k^{2}$, where the term $V(q)$ corresponds to the FT of the scattering potential. For triangular defects like those shown in Ref. 33 and usually found on TI surfaces, the FT corresponds to a flower-like shape, with arms pointing along the $\overline{\Gamma M}$ direction. The length of each arm is inversely proportional to the spatial extension of the defects and amounts to approximately $3 \mathrm{~nm}^{-1}$, a result that does not depend on energy. Both considerations are consistent with our experimental findings and those reported in Ref. 33 and explain why only $\mathbf{q}_{3}$ is clearly visible at $-300 \mathrm{meV}$. At such an 
energy, the above-mentioned pockets becomes ill defined, with bulk states showing a continuum along every direction. Consequently, $\mathbf{q}_{1}$ and $\mathbf{q}_{2}$ vanish since the superposition of waves with various wavelengths smears out the interference pattern.

In summary, we provided a scanning tunneling spectroscopy study of the topological insulator $\mathrm{Bi}_{2} \mathrm{Te}_{3}$. We experimentally obtained the energy dispersion relation for the topological states and visualized the breakdown of their linear dispersion relation, which takes place at an energy of about $690 \mathrm{meV}$ above the Dirac point. Below the Fermi level two distinct scattering channels have been identified. Their orientation and length, consistent with ab initio calculations, reflect the chiral spin texture found in bulk states when they are close in energy and momentum to the resonances of the spin-polarized topological surface states. Our results thus provide evidence that spin-related effects need to be taken into account for bulk states of topological insulators.

We acknowledge support from the Deutsche Forschungsgemeinschaft within SPP 1666 (Grant No. BO1468/21-1), the Ministry of Education and Science of the Russian Federation (State Task No. 2.8575.2013), and the Federal Targeted Program "Scientific and scientific-pedagogical personnel of innovative Russia in 2009-2013" (Grant No. 14B37.21.1164). Calculations were performed on the SKIF-Cyberia supercomputer of Tomsk State University.
*Corresponding author: paolo.sessi@physik.uni-wuerzburg.de

${ }^{1}$ D. Hsieh, D. Qian, L. Wray, Y. Xia, Y. S. Hor, R. J. Cava, and M. Z. Hasan, Nature (London) 452, 970 (2008).

${ }^{2}$ Y. L. Chen, J. G. Analytis, J.-H. Chu et al., Science 325, 178 (2009).

${ }^{3}$ M. Z. Hasan and C. L. Kane, Rev. Mod. Phys. 82, 3045 (2010).

${ }^{4}$ Y. L. Chen, J.-H. Chu, J. G. Analytis et al., Science 329, 659 (2010).

${ }^{5}$ M. Bianchi, R. C. Hatch, J. Mi, B. B. Iversen, and P. Hofmann, Phys. Rev. Lett. 107, 086802 (2011).

${ }^{6}$ H. M. Benia, C. Lin, K. Kern, and C. R. Ast, Phys. Rev. Lett. 107, 177602 (2011).

${ }^{7}$ T. Valla, Z.-H. Pan, D. Gardner, Y. S. Lee, and S. Chu, Phys. Rev. Lett. 108, 117601 (2012).

${ }^{8}$ S. Y. Xu, M. Neupane, C. Liu et al., Nat. Phys. 8, 616 (2012).

${ }^{9}$ A. H. Castro Neto, F. Guinea, N. M. R. Peres, K. S. Novoselov, and

A. K. Geim, Rev. Mod. Phys. 81, 109 (2009).

${ }^{10}$ S.-Y. Xu, Y. Xia, L. A. Wray et al., Science 332, 560 (2011).

${ }^{11}$ M. König, S. Wiedmann, C. Brüne, A. Roth, H. Buhmann, L. W. Molenkamp, X.-L. Qi, and S.-C. Zhang, Science 318, 766 (2007).

${ }^{12}$ P. Roushan, J. Seo, C. V. Parker, Y. S. Hor, D. Hsieh, D. Qian, A. Richardella, M. Z. Hasan, R. J. Cava, and A. Yazdani, Nature (London) 460, 1106 (2009).

${ }^{13}$ T. Zhang, P. Cheng, X. Chen et al., Phys. Rev. Lett. 103, 266803 (2009).

${ }^{14}$ I. Zutic, J. Fabian, and S. Das Sarma, Rev. Mod. Phys. 76, 323 (2004).

${ }^{15}$ L. Fu and C. L. Kane, Phys. Rev. Lett. 100, 096407 (2008).

${ }^{16}$ X.-L. Qi, R. D. Li, J. D. Zang, and S.-C. Zhang, Science 323, 1184 (2009).

${ }^{17}$ P. E. Blöchl, Phys. Rev. B 50, 17953 (1994).
${ }^{18}$ G. Kresse and J. Furthmüller, Phys. Rev. B 54, 11169 (1996).

${ }^{19}$ G. Kresse and D. Joubert, Phys. Rev. B 59, 1758 (1999).

${ }^{20}$ J. P. Perdew, K. Burke, and M. Ernzerhof, Phys. Rev. Lett. 77, 3865 (1996).

${ }^{21}$ D. D. Koelling and B. N. Harmon, J. Phys. C 10, 3107 (1977).

${ }^{22}$ Y. Jiang, Y. Y. Sun, M. Chen et al., Phys. Rev. Lett. 108, 066809 (2012).

${ }^{23}$ Z. Alpichshev, J. G. Analytis, J.-H. Chu, I. R. Fisher, Y. L. Chen, Z. X. Shen, A. Fang, and A. Kapitulnik, Phys. Rev. Lett. 104, 016401 (2010)

${ }^{24}$ M. F. Crommie, C. P. Lutz, and D. M. Eigler, Nature (London) 363, 524 (1993).

${ }^{25}$ J. I. Pascual, G. Bihlmayer, Yu. M. Koroteev et al., Phys. Rev. Lett. 93, 196802 (2004).

${ }^{26}$ J. E. Hoffman, K. McElroy, D.-H. Lee, K. M Lang, H. Eisaki, S. Uchida, and J. C. Davis, Science 297, 1148 (2002).

${ }^{27}$ L. Fu, Phys. Rev. Lett. 103, 266801 (2009).

${ }^{28}$ J. Henk, M. Flieger, I. V. Maznichenko, I. Mertig, A. Ernst, S. V. Eremeev, and E. V. Chulkov, Phys. Rev. Lett. 109, 076801 (2012).

${ }^{29}$ S. Das Sarma, S. Adam, E. H. Hwang, and E. Rossi, Rev. Mod. Phys. 83, 407 (2011).

${ }^{30}$ T. Fang, A. Konar, H. Xing, and D. Jena, Appl. Phys. Lett. 91, 092109 (2007).

${ }^{31}$ D. Hsieh, Y. Xia, D. Qian et al., Phys. Rev. Lett. 103, 146401 (2009).

${ }^{32}$ L. Simon, C. Bena, F. Vonau, M. Cranney, and D. Aubel, J. Phys. D 44, 464010 (2011).

${ }^{33}$ S. Kim, M. Ye, K. Kuroda et al., Phys. Rev. Lett. 107, 056803 (2011). 